\title{
Exploiting natural processes to reduce the need for petroleum-based fertilizers: The role of bacterial chemotaxis in nitrogen fixation of leguminous plants
}

\author{
Julia Sheehan
}

\section{$\underline{\text { Introduction }}$}

Nitrogen is one of the most important nutrients for plant survival, second only to water (Crop Nutrition, 2013). Therefore, it is vital to have a sufficient amount of nitrogen available in soil to produce healthy crops. Nitrogen can be naturally supplied to soil through a process called biological nitrogen fixation. This is a process where soil diazotrophs, specifically rhizobia, form a symbiotic relationship with leguminous plants in order to fix atmospheric nitrogen into ammonia. Once released into the soil, ammonia is a molecule that can be taken up by all plants through the vascular system (Crop Nutrition, 2013)

In a majority of crop production processes, nitrogen is supplied in bulk through the addition of fertilizer. Fertilizers are manufactured using heavy amounts of fossil fuels and can cause lasting harm to the soil through nutrient imbalance and soil acidification. Therefore, at a time when global soil quality has significantly diminished and fossil fuel consumption must be limited, it is important to understand and optimize biological nitrogen fixation in order to reduce the need for petroleum-based fertilizer (Food and Agriculture Organization, 2008).

In order to fix atmospheric nitrogen, the symbiosis process is initiated through a specific cycle of signaling and interactions between the host legume and rhizobia. These signals induce physiological changes in both species and direct the rhizobia to the legume root. The rhizobia enter the cortical root cells at the base of developing root hairs to form nodules. These nodules resemble bulb-like structures on the outer layer of the root and act as vesicles where the 
atmospheric nitrogen is fixed and transported through the legume vascular system (Bergman, 1990).

This study focuses on the way in which the rhizobia are directed towards the host legume. Experiments have shown that the movement of the bacteria is governed through a mechanism called chemotaxis (Wadhams, 2004). Chemotaxis is a physical motile response of bacteria to environmental signals and concentration gradients. It occurs when signals from the environment bind with receptor molecules of the rhizobia and alters the swimming pattern to bias their direction towards the source. This chemotactic behavior of the rhizobia has been shown to be crucial for effective movement, nodulation, and nitrogen fixation of the surrounding legumes (Bergman, 1990, Caetano-Anollés, 1988, \& Soby, 1983). In the future, it could be possible to engineer this chemotactic response of rhizobia in order to optimize biological nitrogen fixation.

There is currently a gap in the understanding of the mechanism by which chemotactic rhizobia move through unsaturated soil. Therefore, the first step toward engineering this behavioral response is to understand exactly what is happening in the soil and how the bacteria are moving to the plant root. It is theorized that the rhizobia require a continuous liquid pathway for their movement. The objective of this research is to analyze the degree that rhizobia can nodulate the host root in both saturated and unsaturated water systems. It is hypothesized that results will show consistent nodulation as moisture levels are decreased until a percolation threshold is reached, at which point continuous liquid pathways no longer exist and nodulation can no longer occurs (Stauffer, 1985). 


\section{Materials and Methods}

General Setup

A rhizotron system has been designed to analyze legume growth and nodulation. The host legume used was Vigna unguiculata, commonly known as the cowpea. The cowpeas were inoculated with Bradyrhizobium Japonicum via N-Dure, a peat-rhizobia mixture from Verdesian Life Sciences. N-Dure is manufactured for inoculation of cowpeas and peanuts, and has a specified rhizobium content of $2 \times 10^{8} \mathrm{CFU} / \mathrm{g}$.

The rhizotron system consists of a $120 \mathrm{~mm}$ square petri dish containing a 100 micron sheet of mesh over a slab of rockwool. The mesh prevents root growth into the media, which created a two dimensional $144 \mathrm{~cm}^{2}$ observation area to assess root and nodule development. The top and bottom edges of the petri dish are open in order to provide growth space for the plant, as well as sufficient ventilation. The seed was transplanted onto the mesh, approximately an inch below the top ventilation edge. Figure 1 demonstrates the rhizotron design with a cowpea grown for 9 days.

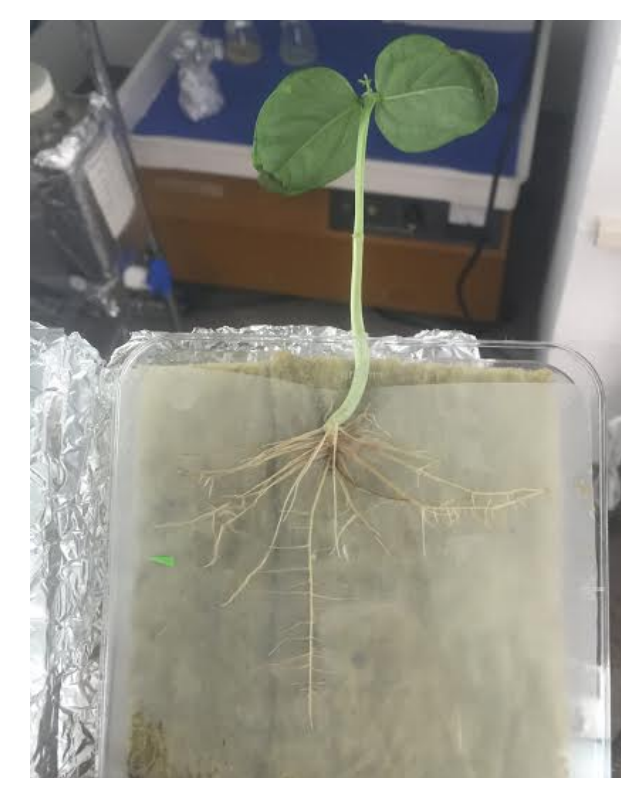

Figure 1: Rhizotron set-up. 
In order to regulate external conditions on cowpea development, growth lamps with 60 watt Sylvania Daylight bulbs were used. Rhizotrons were covered with aluminum foil to simulate darkened soil conditions and encourage light contact solely on leaf surfaces.

The objective of this work was to understand the influence of moisture content, so it was important to be able to control water added to the rhizotron. An irrigation hose design was developed for the rhizotrons, in order to provide uniform watering conditions and have minimal disturbances to bacterial location. Figure 2 depicts the irrigation system, which consisted tubing that ran the length of the rhizotron, and had release holes every $0.15 \mathrm{inch}$. Syringes were used to pump the water into one end of the hose and plug the other, which forced the inputted water to exit the tubing through the release holes along the rhizotron.

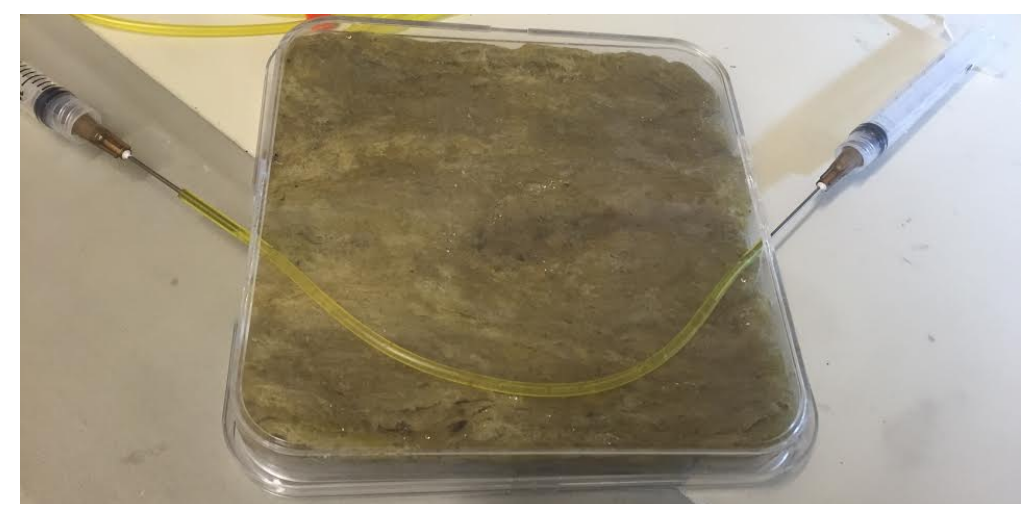

Figure 2: Irrigation watering system.

The B. Japonicum was inoculated into the rhizotron via a sprinkle method, in which the solid peat mixture was directly applied to the system. In designing this experiment, three methods for introducing the rhizobia were compared: a dilute slurry $(1 \mathrm{~g}$ peat in $20 \mathrm{~mL})$, a concentrated slurry $(1 \mathrm{~g}$ peat in $5 \mathrm{~mL})$, and the solid mixture $(1 \mathrm{~g}$ peat $)$ sprinkled onto the system. The dilute slurry hindered root growth since cowpeas are drought resistant and cannot survive in over-watered conditions. The concentrated slurry and sprinkle methods promoted similar nodule 
formation, so the sprinkle method was chosen as to not introduce excess water into the controlled water system.

\section{Data Collection}

The occurrence of nitrogen fixation within the rhizotrons was correlated to successful nodule development. Nodulation was expected to occur within a time span of 20 days from inoculation, respectively. Prior to macroscopic nodule observation, it is possible to collect preliminary results via microscopic staining of nodule primordia. Nodule primordia formation can be observed as early as 5 days after inoculation. The root staining process was developed based on a process by Waluyo (2004) that consists of three parts: fixing the roots for 45 minutes with glycerol, clearing the roots for 1 hour with $6 \%$ active sodium hypochlorite, commonly known as Clorox bleach, and staining the roots with methylene blue dye for 20 minutes. The roots were observed under 40x and 100x magnification and the nodule primordia were counted, in order to quantify successful nitrogen fixation. Figure 3 displays the expected microscopic image for a nodule and nodule primordia formed on a soybean root (Waluyo, 2004).

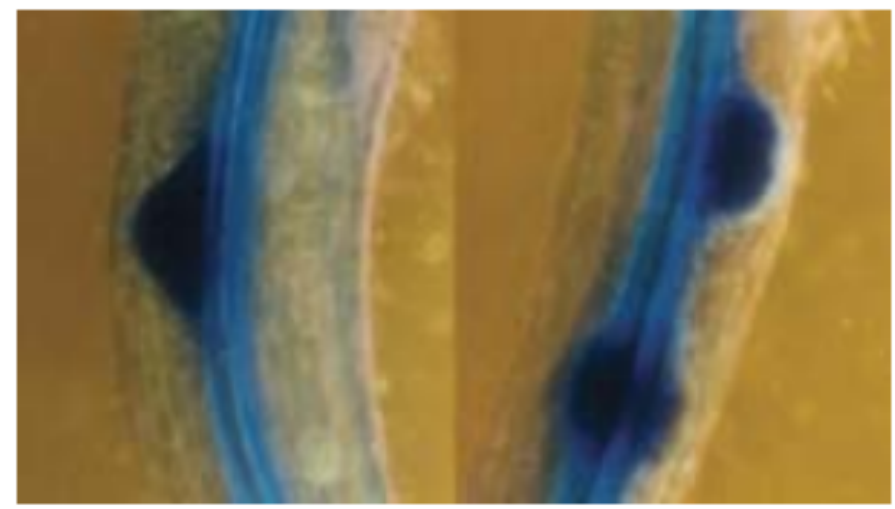

Figure 3: Root (left) and nodule (right) primordia of soybean excised from the plant in a rhizotron experiments and examined under a microscope with magnification of $60 \mathrm{X}$. 
In designing these experiments, Professor Timko from the UVa Biology Department and his laboratory technician, Tatyana Kotova, were consulted. Professor Timko specializes in plant biology and conducts research regarding the genetic mapping of cowpeas.

Experiments

\section{Rhizobia Migration}

The primary question for this project deals with the conditions under which rhizobia can migrate in the soil to the legume root. Therefore, it was important to experimentally demonstrate that bacterial movement was playing a role in nodule formation. This was done by inoculating the rhizotron at the bottom edge of the rhizotron and recording where nodulation occurred, as depicted in figure 4.

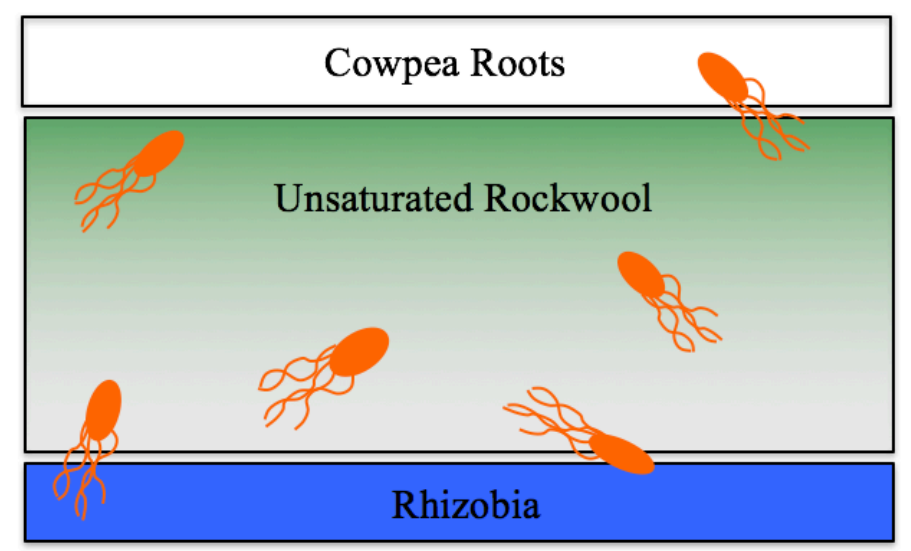

Figure 4: Schematic for chemotactic migration experiment.

\section{Water Effect}

Corresponding to the primary question for this project, and experiment was run to assess the effect of water on chemotaxis and nodule formation. Cowpeas were grown under conditions of $1,3,5$, and $7 \mathrm{~mL}$ of water every 3 days, with two rhizotrons for each volume. 


\section{$\underline{\text { Results and Discussion }}$}

The rhizobia migration experiment demonstrated that nodule formation occurred along a wide area of the root, including early stages of the taproot. Figure 5 demonstrates the zone of the taproot where nodules formed zoomed in to the microscopic view.
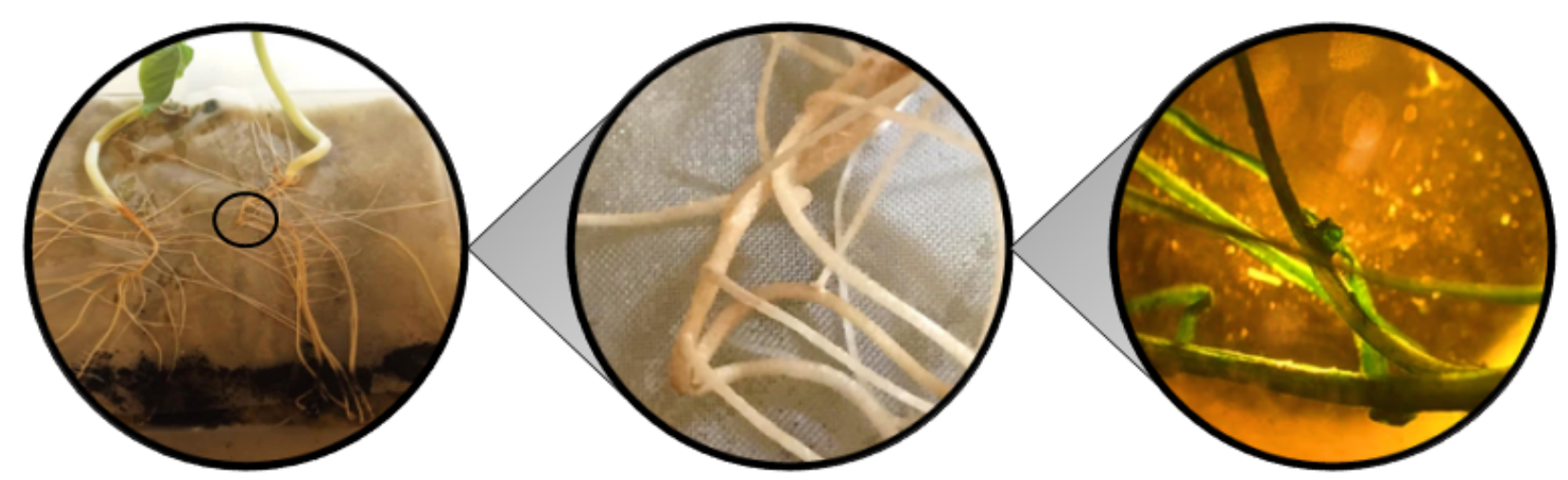

Figure 5: Nodule formed at on early growth stage of the taproot. Zoomed in from rhizotron scale (left) to microscope magnification of 40x (right).

Nodule formation is a time sensitive process because the rhizobia must be able to reach the root hairs as they are developing in order to initiate root infection. Therefore, it was theorized that if nodules formed on the early growth stages of the taproot, it was an indication that the bacteria had moved via directed migration, thus chemotaxis had played a role. This assumption was made because the bacteria would not be able to randomly move to this region of the taproot in the time available for nodulation. The locations where nodules developed on the early taproot, including that shown in figure 5, suggest that chemotaxis is occurring in the rhizotrons in order for nodulation and nitrogen fixation to occur.

The water effect experiment indicated that water does influence nodule formation, and thus nitrogen fixation. The rhizotrons watered with 5 and $7 \mathrm{~mL}$ demonstrated sufficient nodule and nodule primordia formation, as both averaged 8 forming nodules. However, after 20 days the 
rhizotron watered with $1 \mathrm{~mL}$ did not shown any sign of nodule development, and the rhizotron watered with $3 \mathrm{~mL}$ only developed 1 nodule primordia. These results demonstrate that, between 3 and $5 \mathrm{~mL}$, a change is occurring within the system that is inhibiting bacterial movement, and in turn nodulation. This supports the hypothesis that a percolation threshold exists, at which the rhizobia can no longer migrate to the root and induce nitrogen fixation.

\section{Ongoing Work}

Since it has been observed that water content does impact nodulation, the next step in this project is to quantify the moisture content at which rhizobia migration is hindered. A Buchner funnel tensiometer is being designed in order to control the water content in certain pore sizes based on hydrostatic pressure. The tensiometer consists of a Buchner funnel connected by hosing to a burette, similar to the schematic in figure 6 . The intent is to change the height of water in the burette, and an increase in height will create a suction force that will cause smaller pores to empty.

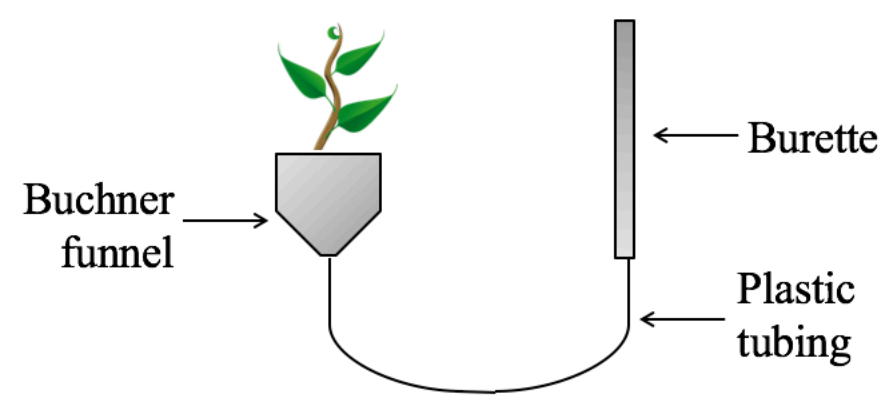

Figure 6: Schematic of tensiometer apparatus.

From this experimental design, it will be possible to identify the percolation threshold for the system. Along with the hydroponic rockwool, a variety of soil types with varying porous characteristics will be tested. The limiting moisture contents that enable bacterial migration and 
nitrogen fixation can then be compared to current climate conditions. This will provide insight into whether it is possible to optimize the biological nitrogen fixation process, or if soil conditions do not promote this process and require research into an alternative sustainable source of nitrogen.

\section{$\underline{\text { Citations }}$}

Bergman, K. (1990, January). The role of bacterial chemotaxis in initiation of rhizobium legume symbiosis for nitrogen-fixation. Journal of Chemical Ecology, 16(1), 114-115.

Caetano-Anollés, G., Wall, L. G., De Micheli, A. T., Macchi, E. M., Bauer, W. D., \& Favelukes, G. (1988). Role of motility and chemotaxis in efficiency of nodulation by Rhizobium meliloti. Plant Physiology, 86(4), 1228-1235.

Food and Agricultural Organization (2008). Retrieved November 26, 2015, from http://www.fao.org/soils-portal/soil-degradation-restoration/en/

Nitrogen. (2013) Retrieved November 25, 2015, from http://www.cropnutrition.com/efu-nitrogen\#additives-for-nitrogen-fertilizers

Soby, S., \& Bergman, K. (1983). Motility and chemotaxis of Rhizobium meliloti in soil. Applied and environmental microbiology, 46(5), 995-998.

Stauffer, D. (1985). Introduction to percolation theory. London: Taylor \& Francis.

Wadhams, G. H., \& Armitage, J. P. (2004). Making sense of it all: bacterial chemotaxis. Nature Reviews Molecular Cell Biology, 5(12), 1024-1037.

Waluyo, S. H., \& Lie, T. A. (2013). Effect of phosphate on nodule primordia of soybean (Glycine max Merrill) in acid soils in rhizotron experiments. Indonesian Journal of Agricultural Science, 5(2). 